\title{
Focal shift in optical waves with off-axis focus
}

\author{
Carlos J. Zapata-Rodríguez*, Genaro Saavedra, Manuel Martínez-Corral \\ Department of Optics, University of Valencia, E-46100 Burjassot, Spain
}

Received 8 June 2002; accepted 27 November 2002

\begin{abstract}
We present a formulation for a suitable description of the focal shift in optical waves that have an off-axis focus. This shift that is primarily produced along the chief axis is given in terms of the focal distance and depends only on a parameter that is named as the generalized Fresnel number. Any non-uniform, either truncated and non-apertured optical beam with off-axis focus may be considered.

(c) 2002 Elsevier Science B.V. All rights reserved.
\end{abstract}

PACS: 42.25.Fx; 42.25.Bs

Keywords: Diffraction; Wave propagation

\section{Introduction}

It is well-known that when a monochromatic, uniform, converging, spherical wave is diffracted by a circular screen, the point of maximum irradiance is not located at the geometrical focus but is somewhat closer to the aperture [1-3]. Li and Wolf [4] showed that the relative focal shift, that is, the ratio of such a shift of the point of maximum irradiance respect to the distance between the geometrical focus and the plane of the aperture, depends only on the Fresnel number of the aperture when viewed from the geometrical focus. The three-dimensional irradiance distribution in low-Fresnel-number focusing systems has been

\footnotetext{
${ }^{*}$ Corresponding author. Fax: +34-96-386-4715.

E-mail address: carlos.zapata@uv.es

(C.J. Zapata-Rodríguez).
}

analytically evaluated [5,6], and experimental evidence of the presence of a focal shift has been reported elsewhere [7,8]. Much more previously it had been found that a very similar phenomenon also exists if the focused beam is an unapertured [9] or a truncated Gaussian laser beam [10], but only after Li and Wolf studies has been recognized that the relative focal shift can be expressed in terms of an effective Fresnel number [11,12].

More sophisticated focusing arrangements have been presented in the literature. For instance, Li [13] studied the axial behavior in the focal region of spherical non-truncated elliptical Gaussian beams. The existence of two irradiance peaks was explained by defining two Gaussian Fresnel numbers associated to the minor and major axes of the elliptical Gaussian beam. In fact, this situation is encountered in other optical systems such as diffracting lenses that generate a great number of foci 
along the optical axis. In this case, a multiple focal shift and focal switch may be presented under low Fresnel numbers associated with every focus [14].

Recently Lü et al. [15-17] have paid a special attention to the problem of the focal shift in windowed Bessel beams, specially Bessel-Gauss beams. In analogy to spherical waves, focused Bessel beams are characterized by a Bessel-Fresnel number. Thus, the point of maximum irradiance along the optical axis is determined approximately in terms of this parameter. However, when this kind of electromagnetic waves are focused they generate a ring pattern in the focal region, thus confirming an oblique focusing behavior $[18,19]$. Consequently, the evaluation of the focal shift in this kind of optical waves must be addressed by evaluating the displacement of the point of maximum irradiance along a single tilted axis passing through the focal ring. The previous statement agrees with the fact that the irradiance distribution of an off-axis point object in the meridian plane of an optical imaging system of low Fresnel number is deformed along the tilted chief ray [20].

The aim of this paper is to present a simple formulation for a suitable description of the focal shift in electromagnetic waves that have an off-axis focus. Focused, apodized Bessel beams are included since they possess the ability of concentrating light around an off-axis ring-shaped region. For that purpose we derive the off-axis irradiance distribution of such a focused optical beam along the tilted chief axis. In order to achieve the point of maximum irradiance along this axis we follow Martínez et al. [12]. The relative focal shift is then approximately evaluated in terms of a unique parameter, which we call as the generalized Fresnel number as a generalization of a previously defined effective Fresnel number. Finally we apply this new formulation for the evaluation of the relative focal shift to a Bessel-Gauss beam and to a uniform offaxis focused wave.

\section{Off-axis focal shift}

Let us consider an off-axis focusing, monochromatic beam whose amplitude transverse distribution at a given plane, which from now on we will denominate as the reference plane, is described by the function $p\left(x_{0}, y_{0}\right)$. The coherent beam is focused in such a way that the wavefront curvature radius, that is, the distance between the reference plane and the focal plane, is given by $\overline{O F}=f$ (see Fig. 1). For the sake of clarity we will write explicitly the part of the transverse amplitude corresponding to the focusing spherical wavefront, which may be produced by an optical element such as a refracting thin lens, and whose center of curvature is located at the axial point of the focal plane. Note that we consider the off-axis focus is generated as a consequence of the propagating beam characteristics and not by the focusing agent. If we assume a scalar paraxial approximation, which holds for low-angular focused waves, we may evaluate the diffracted field at a given point $P(x, y, z)$ in the focal region by simply using the Fresnel-Kirchhoff diffraction formula [21], giving

$$
\begin{aligned}
U(x, y, z)= & \frac{\exp [\mathrm{i} k(z+f)]}{\mathrm{i} \lambda(z+f)} \iint_{-\infty}^{\infty} \frac{\exp (-\mathrm{i} k f)}{f} \\
& \times \exp \left[-\mathrm{i} \frac{k}{2 f}\left(x_{0}^{2}+y_{0}^{2}\right)\right] p\left(x_{0}, y_{0}\right) \\
& \times \exp \left\{\mathrm { i } \frac { k } { 2 ( z + f ) } \left[\left(x-x_{0}\right)^{2}\right.\right. \\
& \left.\left.+\left(y-y_{0}\right)^{2}\right]\right\} \mathrm{d} x_{0} \mathrm{~d} y_{0},
\end{aligned}
$$

where $\lambda$ is the wavelength of the radiation, $k=2 \pi / \lambda$ and the axial coordinate $z$ is measured from focus, $F$.

As assumed previously, the optical wave has one off-axis focus, which is located at a point of

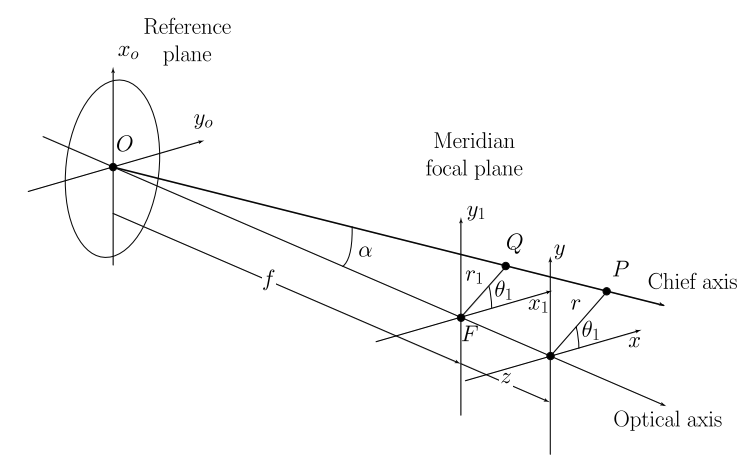

Fig. 1. Schematic diagram of the focusing set-up. 
the focal plane given by $Q\left(x_{1}, y_{1}\right)$. Bessel beams generating a focal ring may be considered by taking one point from this focal region. Thus the region of interest is now addressed over the vicinities of the tilted chief axis, which pass through the axial point of the reference plane, $O$, and the point $Q$. For simplicity, we express Eq. (1) in circular coordinates given by

$x=r \cos \theta, \quad x_{0}=r_{0} \sin \theta_{0}$,

$y=r \sin \theta, \quad y_{0}=r_{0} \cos \theta_{0}$,

where the Cartesian coordinates $\left(x_{0}, y_{0}\right)$ have been appropriately written in order to ease the calculation. The points of the focal volume belonging to the chief axis may be described in terms of the azimuthal coordinate

$\theta_{1}=\arctan \frac{y_{1}}{x_{1}}$,

and the equation

$\frac{r}{z+f}=\frac{r_{1}}{f} \approx \sin \alpha$,

where $r_{1}=\sqrt{x_{1}^{2}+y_{1}^{2}}$ and we have introduced a low-angular approximation, which is valid in the paraxial regime. Now from Eq. (1) we may write the irradiance distribution along the chief axis given by Eqs. (3) and (4) in circular coordinates as

$$
\begin{aligned}
I(z)= & {\left[\frac{2 \pi}{\lambda f(z+f)}\right]^{2} \mid \int_{0}^{\infty} t\left(r_{0}\right) } \\
& \times\left.\exp \left[-\mathrm{i} 2 \pi \frac{z}{2 f \lambda(z+f)} r_{0}^{2}\right] r_{0} \mathrm{~d} r_{0}\right|^{2},
\end{aligned}
$$

where

$$
\begin{aligned}
t\left(r_{0}\right)= & \frac{1}{2 \pi} \int_{-\pi}^{\pi} p_{r}\left(r_{0}, \theta_{0}\right) . \\
& \times \exp \left[-\mathrm{i} 2 \pi r_{0} \frac{\sin \alpha}{\lambda} \sin \left(\theta_{1}+\theta_{0}\right)\right] \mathrm{d} \theta_{0}
\end{aligned}
$$

and

$p_{r}\left(r_{0}, \theta_{0}\right)=p\left(r_{0} \sin \theta_{0}, r_{0} \cos \theta_{0}\right)$.

At this point, it is important to highlight that the one-dimensional function $t\left(r_{0}\right)$ depends on the azimuthal variable $\theta_{1}$ characterizing the chief axis. Azimuthally non-symmetric Bessel beams generally possess more than one chief axis, what implies that $t\left(r_{0}\right)$ may be different for the axes under consideration. However, in the particular case of a radially symmetric beam $p_{r}\left(r_{0}\right)$ we find that the function $t\left(r_{0}\right)$ is independent on the azimuthal angle $\theta_{1}$.

Another significant point is that Eq. (5) is equivalent to that corresponding to the on-axial irradiance distribution of a spherical beam whose focus is at the optical axis. In the latter case, the function $t\left(r_{0}\right)$ stands for the azimuthal average of the amplitude distribution $p_{r}\left(r_{0}, \theta_{0}\right)$ at the reference plane [12], which is assumed to be real and non-negative in order to guarantee that the incoming wave has a focus at the point $F$ in the sense of Geometrical Optics [22]. In our case we must perform a more complicated integral transform given in Eq. (6). Note that both results are in agreement when substituting $\alpha=0$. However, it is important to point out that $t\left(r_{0}\right)$ is imposed to be real, what ensures that the diffracting wave has an off-axis focus at the focal point $Q$ of the chief axis.

In order to find the point of maximum irradiance along the chief axis we should determine the first-derivative zeros of Eq. (5), what usually involves the evaluation of a transcendental equation. Following Martínez-Corral et al. [12] we can avoid this drawback by expanding $I(z)$ into a Taylor series around the point $Q$. As a consequence we may give an analytical expression of the relative focal shift, that is, the ratio of the displacement suffered by the maximum of irradiance along the chief axis respect to the curvature radius of the off-axis focusing beam on the reference plane $f$, as

$\frac{z_{\max }}{f}=-\frac{1}{\pi^{2} N_{\mathrm{g}}^{2}}$,

where we have introduced a new parameter, denominated as the generalized Fresnel number $N_{\mathrm{g}}$ which is written as

$N_{\mathrm{g}}=\frac{\sigma}{\lambda f}$.

In this expression $\sigma$ stands for the standard deviation of the mapped function $t\left(\sqrt{r_{0}}\right)$, i.e.

$\sigma=\left[\frac{M_{2}}{M_{0}}-\left(\frac{M_{1}}{M_{0}}\right)^{2}\right]^{1 / 2}$, 
where

$M_{n}=2 \int_{0}^{\infty} t\left(r_{0}\right) r_{0}^{2 n+1} \mathrm{~d} r_{0}$.

By means of the previously imposed reality to the function $t\left(r_{0}\right)$, reality of $\sigma$ and $M_{n}$ is also found. As we expected the previously defined generalized Fresnel number is inversely proportional to the wavelength and the wavefront curvature radius of the focusing beam, as occurs with the conventional Fresnel number [4,12].

First we point out that the evaluation of the relative focal shift in an off-axis focusing optical wave depends only on the previously defined generalized Fresnel number. This important statement leads to the fact that two different focusing set-ups, independently from the amplitude distribution over the reference plane and the tilt of the chief axis, characterized by the same generalized Fresnel number suffer the same displacement, in relation to the wavefront curvature radius. Additionally, the mentioned dependence follows a negative inverse square law, what implies that the point of maximum irradiance along the chief axis with no exception comes closer to the reference plane for decreasing $N_{\mathrm{g}}$. This effect does not necessarily hold for other focusing geometries such as truncated uniform cylindrical waves [23] and aberrated focusing optical systems [24].

\section{Moments with Besselian kernel}

According to Martínez-Corral et al. [12], Eq. (7) is only valid if $t\left(r_{0}\right)$ is a non-negative real function. First, reality is required since a non-vanishing component in the phase of the function $t\left(r_{0}\right)$ may be associated with the existence of aberrations in the wavefront of the off-axis focusing beam. On the other hand, positivity is also imposed in order to guarantee that the coherent beam has a focus in the sense of Geometric Optics. However, this is a weaker restriction since there exist optical waves that produce off-axis light spots but positivity is not satisfied. In these cases we may also apply the present formalism.

To ensure that the function $t\left(r_{0}\right)$ is real, except for an irrelevant constant phase factor $\exp (-\mathrm{i} \gamma / 2)$, we may impose that $p\left(x_{0}, y_{0}\right) \exp (\mathrm{i} \gamma / 2)$ is an Hermitic function, that is,

$\frac{p_{r}^{*}\left(r_{0}, \theta_{0}+\pi\right)}{p_{r}\left(r_{0}, \theta_{0}\right)}=\exp (\mathrm{i} \gamma), \quad 0 \leqslant \gamma<2 \pi$.

Note that a great variety of off-axis focusing optical beams that we commonly find in the laboratory hold the previous restriction. A tilted uniform plane wave that undergoes the action of a diffraction-limited refracting lens or a propagating Bessel-Gauss beam are clear examples that have encouraged our study. In Section 4 we will apply our formalism to these examples and evaluate the magnitude of the relative focal shift.

Once we have pointed out some of the properties the function $t\left(r_{0}\right)$ should exhibit in order to represent an off-axis focusing beam, we perform the transformation given in Eq. (6). Unfortunately it is possible to find only a few number of cases where such an integral may be analytically performed. However, in order to give an analytical expression of the angular transformation of Eq. (6) we may expand $p_{r}\left(r_{0}, \theta_{0}\right)$ into a circular harmonic series giving

$p_{r}\left(r_{0}, \theta_{0}\right)=\sum_{m=-\infty}^{\infty} p_{m}\left(r_{0}\right) \exp \left(\mathrm{i} m \theta_{0}\right)$,

where

$p_{m}\left(r_{0}\right)=\frac{1}{2 \pi} \int_{-\pi}^{\pi} p_{r}\left(r_{0}, \theta_{0}\right) \exp \left(-\mathrm{i} m \theta_{0}\right) \mathrm{d} \theta_{0}$.

The harmonic expansion in Eq. (12) results of great usefulness either when the number of coefficients contributing to the series is low or when the expansion converges rapidly. By substituting Eq. (12) into Eq. (6) we find

$t\left(r_{0}\right)=\sum_{m=-\infty}^{\infty} p_{m}\left(r_{0}\right) \exp \left(-\mathrm{i} m \theta_{1}\right) J_{m}\left(2 \pi r_{0} \frac{\sin \alpha}{\lambda}\right)$,

where $J_{m}$ is a Bessel function of the first kind, order $m$. Additionally, we have used the relation

$\frac{1}{2 \pi} \int_{-\pi}^{\pi} \exp [\mathrm{i}(m \theta-x \sin \theta)] \mathrm{d} \theta=J_{m}(x)$.

Another important simplification of Eq. (14) may be found by making use of the relationship given 
in Eq. (11) what guarantees reality in the function $t\left(r_{0}\right)$. Thus this restriction can be particularized to the coefficients $p_{m}\left(r_{0}\right)$ yielding

$\frac{p_{-m}^{*}\left(r_{0}\right)}{p_{m}\left(r_{0}\right)}=\exp [\mathrm{i}(\gamma-m \pi)]$

what means that coefficients with negative order may be expressed in terms of those with the same order in absolute value. Therefore, we may express Eq. (14) in a reduced form as

$$
\begin{aligned}
t\left(r_{0}\right)= & p_{0}\left(r_{0}\right) J_{0}\left(2 \pi r_{0} \frac{\sin \alpha}{\lambda}\right)+2 \exp \left(-\mathrm{i} \frac{\gamma}{2}\right) \\
& \times \sum_{m=1}^{\infty}\left|p_{m}\left(r_{0}\right)\right| \cos \left\{m \theta_{1}-\frac{\gamma}{2}-\arg \left[p_{m}\left(r_{0}\right)\right]\right\} \\
& \times J_{m}\left(2 \pi r_{0} \frac{\sin \alpha}{\lambda}\right) .
\end{aligned}
$$

According to Eq. (7) we may evaluate the relative focal shift of an off-axis focusing beam in terms of the generalized Fresnel number, which depends on the radiation wavelength $\lambda$, the curvature radius over the reference plane $f$, and the standard deviation $\sigma$. When using the expansion of Eq. (14) in order to express $t\left(r_{0}\right)$ analytically we find that the moments $M_{n}$ and hence $\sigma$ are also expressed by means of an expansion. By substitution of Eq. (14) into Eq. (10) we find

$$
\begin{aligned}
M_{n} & =2 \int_{0}^{\infty} t\left(r_{0}\right) r_{0}^{2 n+1} \mathrm{~d} r_{0} \\
& =\sum_{m=-\infty}^{\infty} m_{n, m} \exp \left(-\mathrm{i} m \theta_{1}\right), \quad n=0,1,2, \ldots,
\end{aligned}
$$

where

$m_{n, m}=2 \int_{0}^{\infty} p_{m}\left(r_{0}\right) J_{m}\left(2 \pi r_{0} \frac{\sin \alpha}{\lambda}\right) r_{0}^{2 n+1} \mathrm{~d} r_{0}$,

that is, we may express the moments of the function $q(\zeta)$ in terms of a series involving moments of the Fourier coefficients $p_{m}\left(r_{0}\right)$ with Besselian kernel. Finally, by using Eq. (16) we find a symmetry in the coefficients $m_{n, m}$ given by

$\frac{m_{n,-m}^{*}}{m_{n, m}}=\exp (\mathrm{i} \gamma)$,

which may be used in the expansion of Eq. (18).

\section{Examples}

Some examples will allow us to clarify some aspects of the presented formalism. Two common off-axis focusing waves we find in the laboratory are a uniform off-axis converging spherical wave and a Bessel-Gauss beam generated, for example, by an axicon that is illuminated by a spherical Gaussian beam. Let us specifically discuss both examples in detail.

\subsection{Uniform off-axis converging spherical wave}

The three-dimensional irradiance distribution generated by an off-axis point object at the meridian plane of a diffraction-limited optical imaging system of low Fresnel number has been studied by Namikawa [20]. He found a significant deformation along the tilted chief axis and hence no symmetries about the geometrical point image are conserved in this case. If we consider a circular clear exit pupil of radius $a$, the amplitude transverse distribution of a uniform off-axis spherical wave at the reference plane is then written as

$p_{r}\left(r_{0}, \theta_{0}\right)=\exp \left(\mathbf{i k}_{\perp} \mathbf{r}_{0}\right) \operatorname{circ}\left(r_{0} / a\right)$,

where

$\operatorname{circ}(r)= \begin{cases}1, & r \leqslant 1, \\ 0, & \text { otherwise. }\end{cases}$

Note that we have not included a quadratic phase factor that is explicitly written in Eq. (1). In this case both the transverse spatial coordinate $\mathbf{r}_{0}=\left(r_{0} \sin \right.$ $\left.\theta_{0}, r_{0} \cos \theta_{0}\right)$ and the transverse wave number

$\mathbf{k}_{\perp}=2 \pi \frac{\sin \alpha}{\lambda}\left(\cos \theta_{1}, \sin \theta_{1}\right)$

are given as a two-dimensional vector in such a way that the vector $\mathbf{k}_{\perp}$ provides the direction of the off-axis chief ray (see Fig. 1).

In order to evaluate the off-axis irradiance distribution we first evaluate the one-dimensional function $t\left(r_{0}\right)$ by introducing Eq. (21) into Eq. (6) what yields

$t\left(r_{0}\right)=\operatorname{circ}\left(r_{0} / a\right)$.

This transformed amplitude distribution at the reference plane coincides with the azimuthal 
average of that given by a uniform on-axis spherical wave. According to this analogy, we conclude that within the paraxial regime the irradiance distribution along the tilted chief axis of an off-axis spherical wave coincides with that given by an onaxis spherical wave along the optical axis. In terms of the generalized Fresnel number we first substitute Eq. (24) into Eq. (8) giving

$N_{\mathrm{g}}=\frac{1}{\sqrt{12}} \frac{a^{2}}{\lambda f}$

what coincides with both the effective Fresnel number of a uniform spherical wave given by Martínez-Corral et al. [12] and, except for an irrelevant factor $1 / \sqrt{12}$, the Fresnel number of the focusing geometry as given by Li and Wolf [4], as expected.

\subsection{Bessel-Gauss beam}

Bessel beams are of particular interest due to the possibility of producing a diffraction-free pencil of light. However, this kind of waves carry an infinite amount of energy. In order to meet with experimental feasibility we should consider apertured Bessel beams. This is the reason why much attention have been paid to the so-called BesselGauss beams, whose transverse amplitude distribution may be written in the form

$p_{r}\left(r_{0}\right)=J_{0}\left(k r_{0} \sin \alpha\right) \exp \left[-\left(r_{0} / \omega\right)^{2}\right]$.

Optical beams with similar characteristics may be produced with the aid of an axicon that is illuminated by a spherical Gauss beam [25].

The three-dimensional diffraction field may be thought as a superposition of a continuous set of Gaussian beams, whose propagation axes are evenly distributed on the surface of a cone [26]. The axis of the latter coincides with the optical axis and its aperture angle is given by $\alpha$. Consequently, when investigating the focal shift observed in Bessel-Gauss beams we should consider a tilted axis satisfying Eq. (4). The off-axis irradiance distribution given in Eq. (5) is then evaluated in terms of the one-dimensional function $t\left(r_{0}\right)$. However, profiting from the radial symmetry of $p_{r}\left(r_{0}\right)$ and according to Eqs. (13) and (14) it is possible to straightforwardly obtain this function giving
$t\left(r_{0}\right)=J_{0}^{2}\left(k r_{0} \sin \alpha\right) \exp \left[-\left(r_{0} / \omega\right)^{2}\right]$,

which is a non-negative real function, as expected.

The generalized Fresnel number corresponding to a spherical Bessel-Gauss beam is then obtained from Eqs. (9) and (8). Fortunately, we find an analytical expression of the standard deviation $\sigma$ in Eq. (27) giving a generalized Fresnel number of the form

$$
\begin{aligned}
N_{\mathrm{g}}= & \frac{\omega^{2}}{\lambda f}\left(\frac{1}{2}\left[(\beta-2)^{2}-\beta(\beta-3) \frac{I_{1}(\beta / 2)}{I_{0}(\beta / 2)}\right]\right. \\
& \left.-\frac{1}{4}\left[(\beta-2)-\beta \frac{I_{1}(\beta / 2)}{I_{0}(\beta / 2)}\right]^{2}\right)^{1 / 2},
\end{aligned}
$$

where $\beta=k^{2} \omega^{2} \sin ^{2} \alpha$, and $I_{n}$ stands for a modified Bessel function of the first kind, order $n$.

In Fig. 2 it is depicted the generalized Fresnel number, normalized to the previously defined Gaussian-Fresnel number [11] given as $N_{\mathrm{G}}=$ $\omega^{2} / \lambda f$, versus the adimensional coordinate $\beta$ and some important conclusions are obtained from this figure and Eq. (28). First, we may reproduce the result concerning to a spherical Gaussian beam for a vanishing $\beta$. This limit is equivalent to set $\alpha=0$ what means that the Bessel function has no influence on the diffracting field after focalization, then provoking the absence of any tilt over the focus. In this case, the generalized Fresnel number is given by $N_{\mathrm{g}}=\omega^{2} / \lambda f$, which coincides with the Gaussian-Fresnel number. Otherwise, for high values of $\alpha$ within the paraxial approximation we may con-

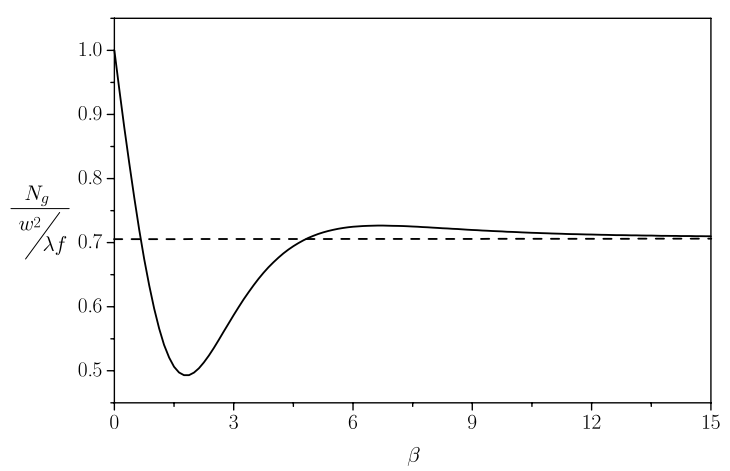

Fig. 2. The generalized Fresnel number, normalized to the Gaussian-Fresnel number, versus the adimensional coordinate $\beta$ for a Bessel-Gauss beam. 
sider that the width of the Gaussian profile is large enough so that the Bessel beam aperturing is weak. In this case holds the inequality $k \sin \alpha \gg \omega^{-1}$ what is equivalent to make the limit $\beta$ tending to infinity. In this case, we find that the generalized Fresnel number is given by

$N_{\mathrm{g}}=\frac{1}{\sqrt{2}} \frac{\omega^{2}}{\lambda f}$.

In this regime we observe that the generalized Fresnel number is nearly independent of the focused field tilt. In other words, the Bessel beam determines the off-axis tilt the wave field suffers in the diffraction process, but not the relative focal shift magnitude. This result is in clear agreement with that previously found for a uniform off-axis focused field, whose generalized Fresnel number is also independent on the off-axis tilt.

\section{Discussion and conclusions}

The focal shift effect, which is a phenomenon observed in spherical beams with long focal distance, appears also in off-axis focused optical waves. The shift is primarily produced along the chief tilt axis, and its magnitude, given in terms of the focal distance, depends only on a parameter that is named as the generalized Fresnel number. Any non-uniform, either truncated or non-apertured optical beam with off-axis focus in the sense of Geometrical Optics may be considered.

Under the scalar paraxial regime it is seen that the irradiance distribution along the chief tilt axis is governed by an integral transform that is equivalent to that in on-axis spherical beams when considering the optical axis. However, first it is necessary to perform an angular transformation over its amplitude transverse distribution, what may be interpreted as an apodizing effect. It is remarkable that this apodizing effect depends on the tilt of the chief axis, and hence the relative focal shift varies according to the location of the off-axis focus under consideration. However, we have shown that this departure in terms of the tilt angle is modest and, for example, spherical beams with a focus at different points of the focal plane provides the same amount of focal shift. Moreover, as oc- curs with windowed Bessel beams the existence of several off-axis foci on the focal plane should enforce the evaluation of the generalized Fresnel number that would be associated with different chief axes characterizing the focusing set-up.

\section{Acknowledgements}

This work was supported by the Plan Nacional I+D+I (Grant DPI2000-0774), Ministerio de Ciencia y Tecnología, Spain. Carlos J. ZapataRodríguez is also grateful for a postdoctoral grant awarded by the Ministerio de Educación, Cultura y Deporte, Spain.

\section{References}

[1] A. Arimoto, Opt. Acta 23 (1976) 245.

[2] J.J. Stamnes, B. Spjelkavik, Opt. Commun. 40 (1981) 81.

[3] J.H. Erkkila, M.E. Rogers, J. Opt. Soc. Am. 71 (1981) 904.

[4] Y. Li, E. Wolf, Opt. Commun. 39 (1981) 211.

[5] Y. Li, E. Wolf, J. Opt. Soc. Am. A 8 (1984) 801.

[6] Y. Li, J. Opt. Soc. Am. A 4 (1987) 1349.

[7] Y. Li, H. Platzer, Opt. Acta 30 (1983) 1621.

[8] G.P. Karman, A. van Duijl, M.W. Beijersbergen, J.P. Woerdman, Appl. Opt. 36 (1997) 8091.

[9] G. Goubau, in: E.C. Jordan (Ed.), Electromagnetic Theory and Antennas Part 2, Macmillan, 1963.

[10] D.A. Holmes, J.E. Korka, P.V. Avizonis, Appl. Opt. 11 (1972) 565.

[11] W.H. Carter, Appl. Opt. 21 (1982) 1989.

[12] M. Martínez-Corral, C.J. Zapata-Rodríguez, P. Andrés, E. Silvestre, J. Opt. Soc. Am. A 15 (1998) 449.

[13] Y. Li, Opt. Commun. 68 (1988) 317.

[14] Y. Li, J. Opt. Soc. Am. A 14 (1997) 1297.

[15] B. Lü, W. Huang, Opt. Commun. 109 (1994) 43.

[16] B. Lü, W. Huang, B. Zhang, F. Kong, Q. Zhai, Opt. Commun. 131 (1996) 223.

[17] B. Lü, W. Huang, J. Mod. Opt. 43 (1996) 509.

[18] Z. Bouchal, J. Wagner, M. Olivik, Opt. Eng. 43 (1995) 1680.

[19] C. Palma, Appl. Opt. 36 (1997) 1116.

[20] T. Namikawa, M. Shibuya, Optik 96 (1994) 93.

[21] J. Goodman, Introduction to Fourier Optics, McGrawHill, Singapore, 1996.

[22] E. Collet, E. Wolf, Opt. Lett. 5 (1980) 264.

[23] C.J. Zapata-Rodríguez, M. Martínez-Corral, P. Andrés, A. Pons, J. Mod. Opt. 46 (1999) 129.

[24] A. Yoshida, T. Asakura, Opt. Commun. 109 (1994) 368.

[25] R.M. Herman, T.A. Wiggings, J. Opt. Soc. Am. A 8 (1991) 932.

[26] M. Santarsiego, Opt. Commun. 132 (1996) 1. 